\title{
Tuned aggregation and film self-assembly of monomethincyanine dyes through variation of their monomer structure
}

\author{
M.M. Sieryk, O.P. Dimitriev \\ V. Lashkaryov Institute of Semiconductor Physics, NAS of Ukraine \\ 41, prospect Nauky, 03680 Kyiv, Ukraine \\ E-mail: serikmykola@gmail.com
}

\begin{abstract}
Formation of condensed films and solution aggregates of four different monomethincyanine dyes have been studied using optical absorption spectroscopy and simulation methods, depending on variation of the dye monomer structure. The structure of molecular dimer as a basic unit for formation of the condensed state was found to be largely dependent on heteroatoms in the dye structure and the presence of end hydrocarbon groups. The above factors mainly determine the mutual position of molecules in the dimer. It has been found that mutual orientation, intermolecular distance and overlap of the adjacent molecules are the major factors influencing absorption spectra of dye aggregates. The dimer geometry that plays the primary role in film nucleation, however, has been shown to undergo changes depending on the temperature conditions or film thickness.
\end{abstract}

Keywords: self-assembly, aggregate, monomethincyanine dyes, absorption spectrum.

doi: https://doi.org/10.15407/spqeo22.01.53

PACS 78.40.Me, 81.16.Dn

Manuscript received 11.01.19; revised version received 29.01.19; accepted for publication 20.02.19; published online 30.03.19.

\section{Introduction}

Self-assembly of molecular systems is driven mostly by competition of molecule-molecule and moleculeenvironment (solvent, substrate, etc.) interactions [1, 2]. Since the above interactions are normally weak and dominating by weak electrostatic and van der Waals forces, the long-range order of the self-assembled system does not normally persist, while the short-range order can undergo significant variations providing rather different geometry of molecular pairs in dimmers that play the role of elementary units to construct the bulk structure [3].

The rate of self-assembly is another factor that influences the final structure of a self-assembled system. The film samples from solution were formed relatively slowly, which is controlled by the rate of solvent evaporation. The films prepared using vacuum evaporation are formed faster through immediate sticking of molecules to the substrate, where the molecules possess rather slow mobility as compared to that in solution, resulting in more loose structure obtained.

One of the main factors that defines the intermolecular interaction is the chemical structure of molecule, which can be tuned to drive the structure of the condensed film overall and to change its physical properties, respectively. Monomethincyanine dyes belong to polymethine dyes serving as convenient model objects that allow one to understand how slight variation in the molecular structure can influence formation of the aggregate or bulk structure [4-7]. These dyes have relatively short length and are stable upon thermal evaporation in vacuum [8]. They can be found in different optoelectronic applications, such as optical switching [9], fluorescent probes [10], photographic imaging [11], etc.

In this work, we study aggregate formation of monomethincyanine bases with unsymmetrical neutral linear structure containing donor and acceptor terminal groups, which can be considered as neutral derivatives produced from the corresponding cationic cyanines with the same $\pi$-elecron system. Similarly to other polymethine dyes, monomethincyanine bases have highly polarizable collective system of $\pi$-electrons and possess high molar absorption coefficient and high hyperpolarizabilities. On the other hand, these dyes, owing to their unsymmetrical structure, have large dipole moments in both ground and excited states [5]. We demonstrate that a slight variation in the dye structure possessing the same chromophore nuclei can lead to significant changes in formation of their condensed films. 


\section{Experimental}

Four types of intraionic (monometine) dyes (bases of asymmetric monometincyanine derivatives of benzo[c, d]indole) were investigated (Fig. 1). The dyes were synthesized at Institute of Organic Chemistry, National Academy of Sciences of Ukraine as described elsewhere [5]. Monomer solutions of these compounds were prepared by dissolving the dye molecules in dioxane. The stock solution had the concentration of $10^{-3} \mathrm{M}$, but optical absorption spectra were recorded for the diluted $\left(10^{-5} \mathrm{M}\right)$ solutions. Dye aggregation was provided by addition of a small amount ( 1 vol.\%) of the dioxane stock solution to the distilled water, where the compounds are not soluble. The spectra of solutions were measured using the Avantes-2048 spectrophotometer and corrected for the pure solvent.

Films were deposited using the VUP-5 vacuum chamber equipped with the optical spectrometer Polytec to control the spectral changes in-situ. Compounds were evaporated at the pressure of $10^{-3} \mathrm{~Pa}$ by using resistive heated silica crucible. Thin films of dyes were fabricated at the deposition rate of $0.1 \ldots 1.6 \mathrm{E} / \mathrm{s}$ onto the cleaned and annealed glass substrates.

Morphology of prepared specimens was investigated with the AFM microscope NanoScope IIIa (Digital Instruments, USA). Molecular simulation was performed with Gaussian 09 and Materials Studio 8.0 (Forcite Module) software by using the density functional method (DFT/B3LYP 6-31+(d,p)) and the method of molecular dynamics (MD).

\section{Results and discussion}

\subsection{Self-assembly of Py}

The structure of Py molecule (Fig. 1) was found to be ideally flat, with a zero torsional angle between the benzo[c, d]indole and pyridine units as described by the simulation results (Table 1). The flat structure of the monomer facilitates formation of almost ideal molecular stacks when forming a dimer (Fig. 2a, Table 1). This stacking or $\mathrm{H}$-aggregate formation should be identified through blue shift in the absorption spectra $[12,13]$. However, it was found an insignificant blue shift of the dimer spectrum relatively the monomer one, by only $\sim 200 \mathrm{~cm}^{-1}$ (Fig. 2b). Moreover, formation of films did not show any clear spectral shift of the absorption band. Instead, the band was broadened, indicating significant disorder in the film structure. The microscopic image confirmed that the film structure had amorphous character, since no formation with a regular shape was found (Fig. 2c).

The contradiction between ability of Py molecules to form ideal short-order within a dimer and failure to form the long-range order can be explained by relatively large distance between the molecules (Table 1). Specifically, the equilibrium distance between the molecules with no hydrogen bonding should be about $3.5 \AA$ [14], while a $3.65 \AA$ Py-Py distance has been found in the dimer. This large distance leads to more relative freedom of molecules separated by this distance and, therefore, to high disorder in the condensed state, respectively.

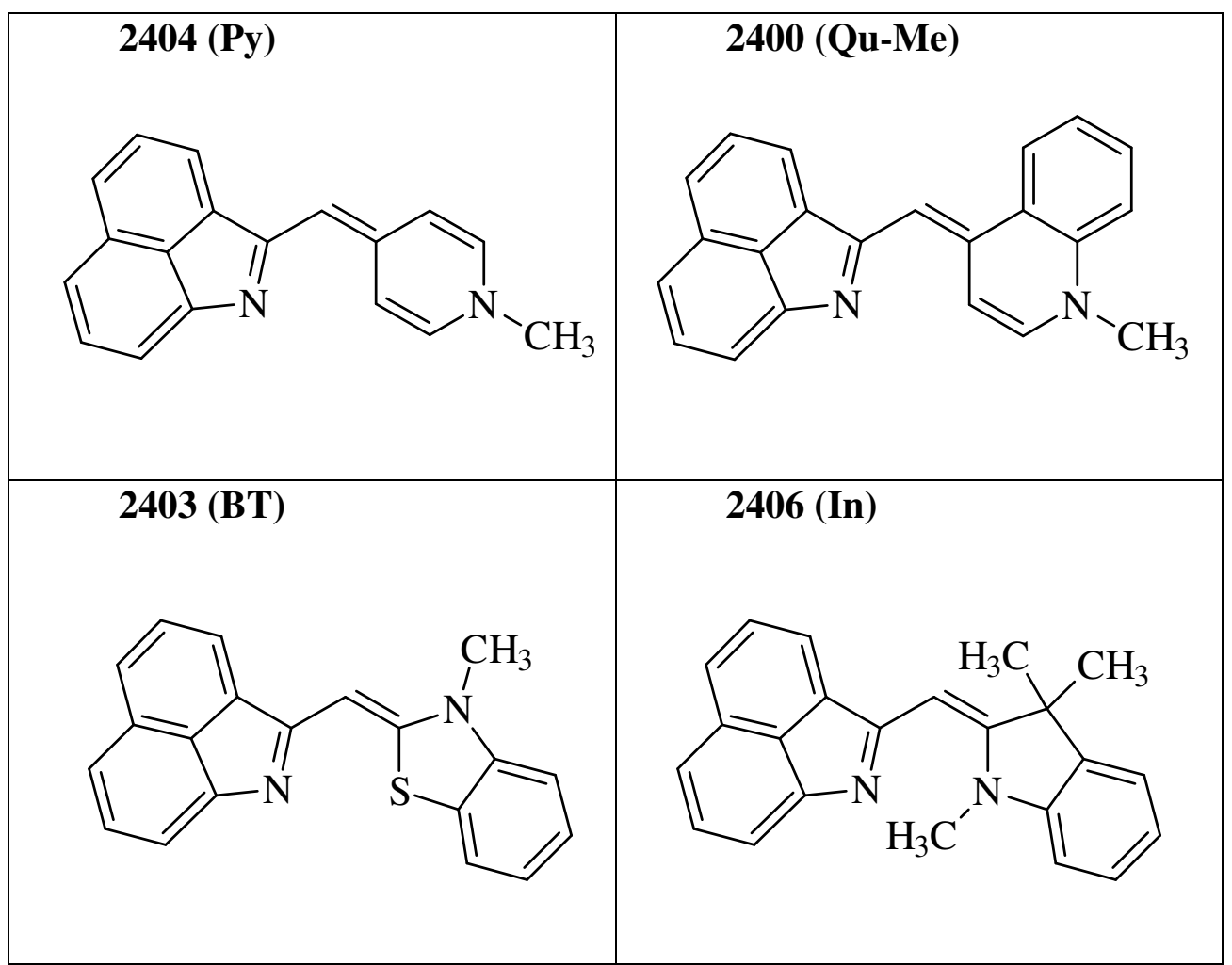

Fig. 1. Chemical structure of the compounds used. 


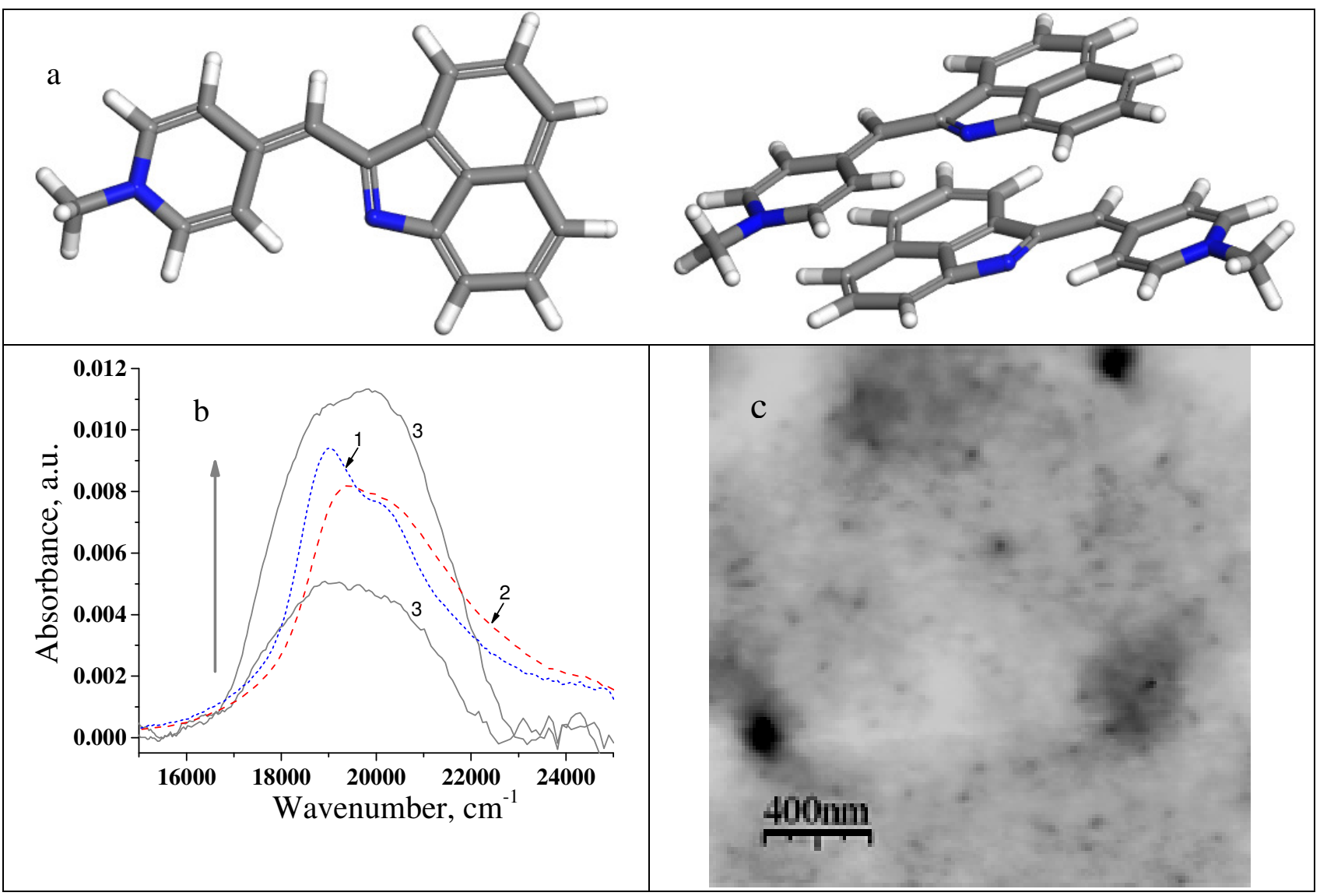

Fig. 2. (a) Simulated structure of Py monomer and dimer, (b) electronic absorption spectra of monomer in dioxane solution (blue, 1), dimer in aqueous solution (red, 2) and evaporated film with increasing thickness (grey, 3), (c) typical AFM image of the film surface.

Table 1. Parameters of Py monomer and dimer obtained as a result of simulation.

\begin{tabular}{|l|c|c|c|}
\hline \multirow{2}{*}{ Physical quantity } & \multirow{2}{*}{ Monomer } & \multicolumn{2}{|c|}{ Dimer } \\
\cline { 3 - 4 } & & Molecule 1 & Molecule 2 \\
\hline Torsion angle & 0 & 0 & 0 \\
\hline Dipole moment & 1.62 & 1.51 & \multicolumn{2}{|c|}{$150^{\circ}(-0.86)$} \\
\hline $\begin{array}{l}\text { Angle between dipole moments of } \\
\text { molecules }(\cos \alpha)\end{array}$ & & \multicolumn{2}{|c|}{0.8} \\
\hline Dipole moment of dimer & & \multicolumn{2}{|c|}{3.65} \\
\hline Distance between molecules $(\AA)$ & & \multicolumn{2}{|c|}{} \\
\hline
\end{tabular}

\subsection{Self-assembly of $Q u-M e$}

The structure of Qu-Me molecule (Fig. 1) is somewhat different from that of Py by the presence of pyridine at the quinoline unit only. However, this change leads to significant difference in the aggregate behavior. First, it was found that the dimer of $\mathrm{Qu}-\mathrm{Me}$ is also ideally flat, with the zero torsional angle between the benzo[c, d]indole and quinoline nuclei as described by the simulation results (Table 2), however, the intermolecular distance is notably shorter and close to $3.2 \AA$. The flat structure of the monomer implies formation of almost ideal molecular stacks in the dimer as well (Fig. 3a, Table 2). And this stacking or $\mathrm{H}$ aggregate formation is really identified through blue shift in the absorption spectra (Fig. 3b). Specifically, the monomer absorption in the solution shows an electronic 0-0 transition at $\sim 15870 \mathrm{~cm}^{-1}$ accompanied by vibronic transitions separated by $\sim 1200 \mathrm{~cm}^{-1}$, whereas the absorption spectrum of the dimer is blue shifted in respect to the monomer absorption by $\sim 2000 \mathrm{~cm}^{-1}$, which indicates that the molecules tend to form $\mathrm{H}$-aggregates. 




Fig. 3. (a) Simulated structure of Qu-Me monomer and dimer, (b) electronic absorption spectra of monomer in dioxane solution (blue, 1), dimer in aqueous solution (red, 2) and evaporated film which was monitored during growth in-situ (grey, 3) and then relaxed for 10 minutes after evaporation (green, 4), (c) typical AFM image of the film surface.

Table 2. Parameters of Qu-Me monomer and dimer obtained as a result of simulation.

\begin{tabular}{|c|c|c|c|}
\hline \multirow{2}{*}{ Physical quantity } & \multirow{2}{*}{ Monomer } & \multicolumn{2}{|c|}{ Dimer } \\
\hline & & Molecule 1 & Molecule 2 \\
\hline Torsion angle & $180^{\circ}$ & $178^{\circ}$ & $178^{\circ}$ \\
\hline Dipole moment & 2.24 & 1.15 & 1.16 \\
\hline $\begin{array}{l}\text { Angle between dipole moments of } \\
\text { molecules }(\cos \alpha)\end{array}$ & & \multicolumn{2}{|c|}{$175^{\circ}(-0.99)$} \\
\hline Dipole moment of dimer & & \multicolumn{2}{|c|}{0.09} \\
\hline Distance between molecules $(\AA)$ & & \multicolumn{2}{|c|}{3.2} \\
\hline
\end{tabular}

However, the Qu-Me film growth on the glass substrate demonstrates a totally different trend. The film spectrum is red shifted in respect to the monomer absorption, which corresponds to formation of $\mathrm{J}$ aggregates in the condensed film [14]. The marginal signs of $\mathrm{H}$-aggregates can be seen only as a broadening from the high-energy side of the film spectrum (Fig. 3b). The observed difference between the aggregate structures in solutions and films can be explained by different dyesolvent and dye-substrate interactions. Although in both cases the dye environment is hydrophilic, in the case of solution the dimers are isolated in a disordered hydrophilic environment, while in the condensed film the molecules tend to be arranged in more extended aggregates, which structure can be dictated by the crystalline order. Indeed, the AFM image of the film
(Fig. 3c) indicates the presence of species with quasiregular shapes, which is characteristic of crystallite formation. Similar result was obtained in ref. [15]. Thus, the flat molecular structure of Qu-Me allows the molecules to approach relatively small distance to each other (Table 1) and leads to formation of two types of aggregates, either $\mathrm{H}$ - or J-type. It is interesting to note that J-aggregates can be transformed to $\mathrm{H}$-aggregates through film relaxation (green curve 4 in Fig. 3b).

\subsection{Self-assembly of BT}

The equilibrium structure of BT molecule (Fig. 1) is not ideally flat and displays a certain torsion angle between the donor and acceptor units (Table 3). Nevertheless, 


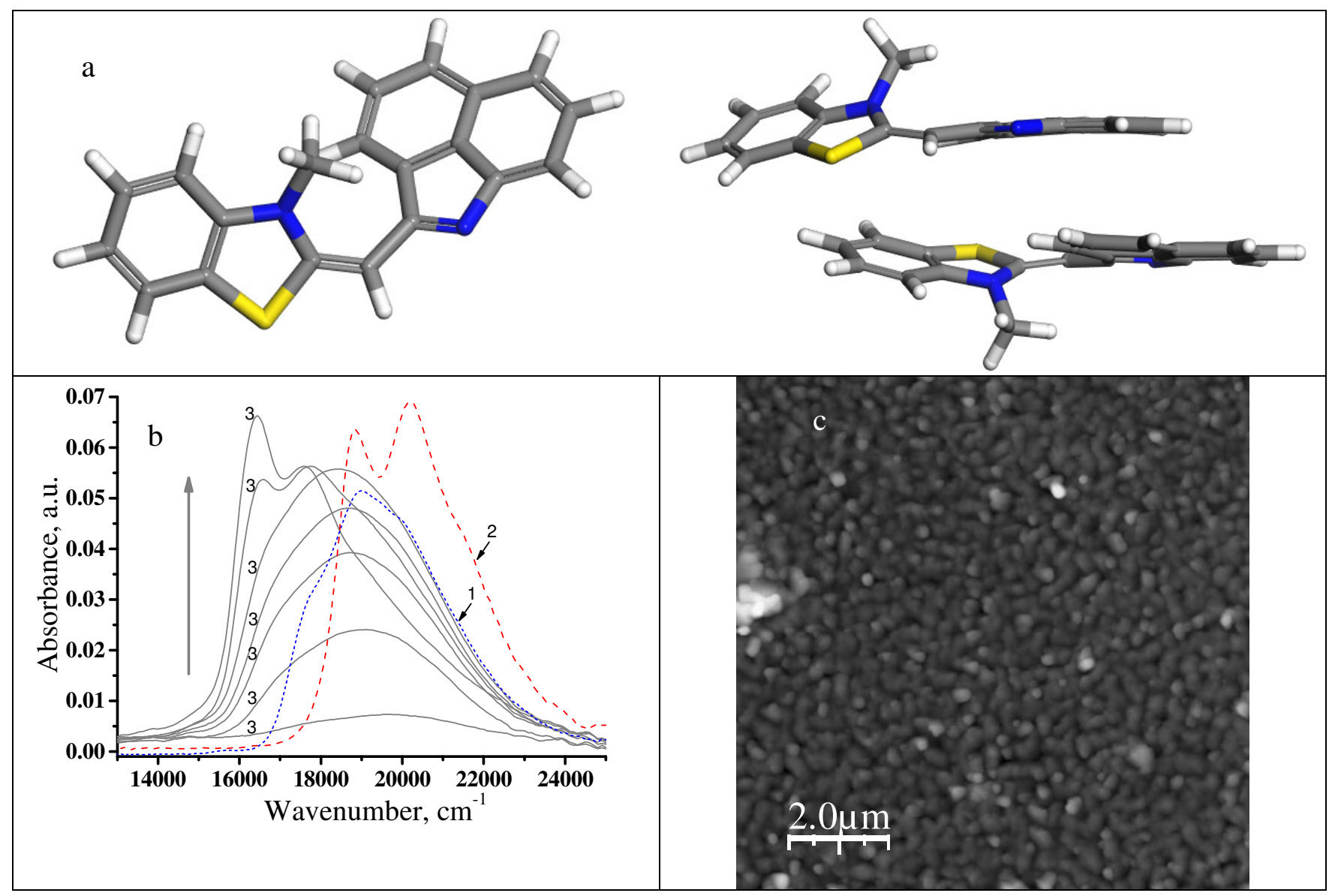

Fig. 4. (a) Simulated structure of BT monomer and dimer, (b) electronic absorption spectra of monomer in dioxane solution (blue, 1), dimer in aqueous solution (red, 2) and evaporated film monitored during growth in-situ (grey, 3), (c) typical AFM image of the film surface.

Table 3. Parameters of BT monomer and dimer obtained using simulation.

\begin{tabular}{|c|c|c|c|}
\hline \multirow{2}{*}{ Physical quantity } & \multirow{2}{*}{ Monomer } & \multicolumn{2}{|c|}{ Dimer } \\
\hline & & Molecule 1 & Molecule 2 \\
\hline Torsion angle & $-11.5^{\circ}$ & $-15.5^{\circ}$ & $-12.2^{\circ}$ \\
\hline Dipole moment & 3.41 & 3.19 & 3.41 \\
\hline $\begin{array}{l}\text { Angle between dipole moments of } \\
\text { molecules }(\cos \alpha)\end{array}$ & & \multicolumn{2}{|c|}{$122^{\circ}(-0.54)$} \\
\hline Dipole moment of dimer & & \multicolumn{2}{|c|}{2.95} \\
\hline Distance between molecules $(\AA)$ & & \multicolumn{2}{|c|}{3.35} \\
\hline
\end{tabular}

according to the simulation results, the intermolecular distance in the BT dimer is relatively small, i.e., $3.35 \AA$, which leads to the significant difference in the dimer spectrum as compared to that of monomer one (Fig. 4). Specifically, the spectral features of the BT dimer were characteristic of $\mathrm{H}$-aggregate. However, the BT film again demonstrated a totally different trend, similar to that observed in Qu-Me films, i.e., the film spectrum was red-shifted in respect to the monomer absorption, which corresponds to formation of J-aggregates in the condensed film. This difference between the aggregate structures in solutions and films can be again explained by different dye-solvent and dye-substrate interactions, where in the condensed film the molecules tend to be arranged in more extended aggregates, which structure is dictated by the crystalline order. The AFM image of the film (Fig. 4c) indicated the structure composed of features of several hundreds of nanometers in size, suggesting formation of crystallites. That means that the molecular structure of BT allows the molecules to form two types of aggregates, either $\mathrm{H}$ - or J-type, similar to those of Qu-Me molecules above.

\subsection{Self-assembly of In}

The structure of the In molecule is different from that of BT by replacement of the sulfur atom in BT chromophore with the carbon atom, to which two additional methyl groups are attached (Fig. 1). The presence of additional side groups may provide a certain 


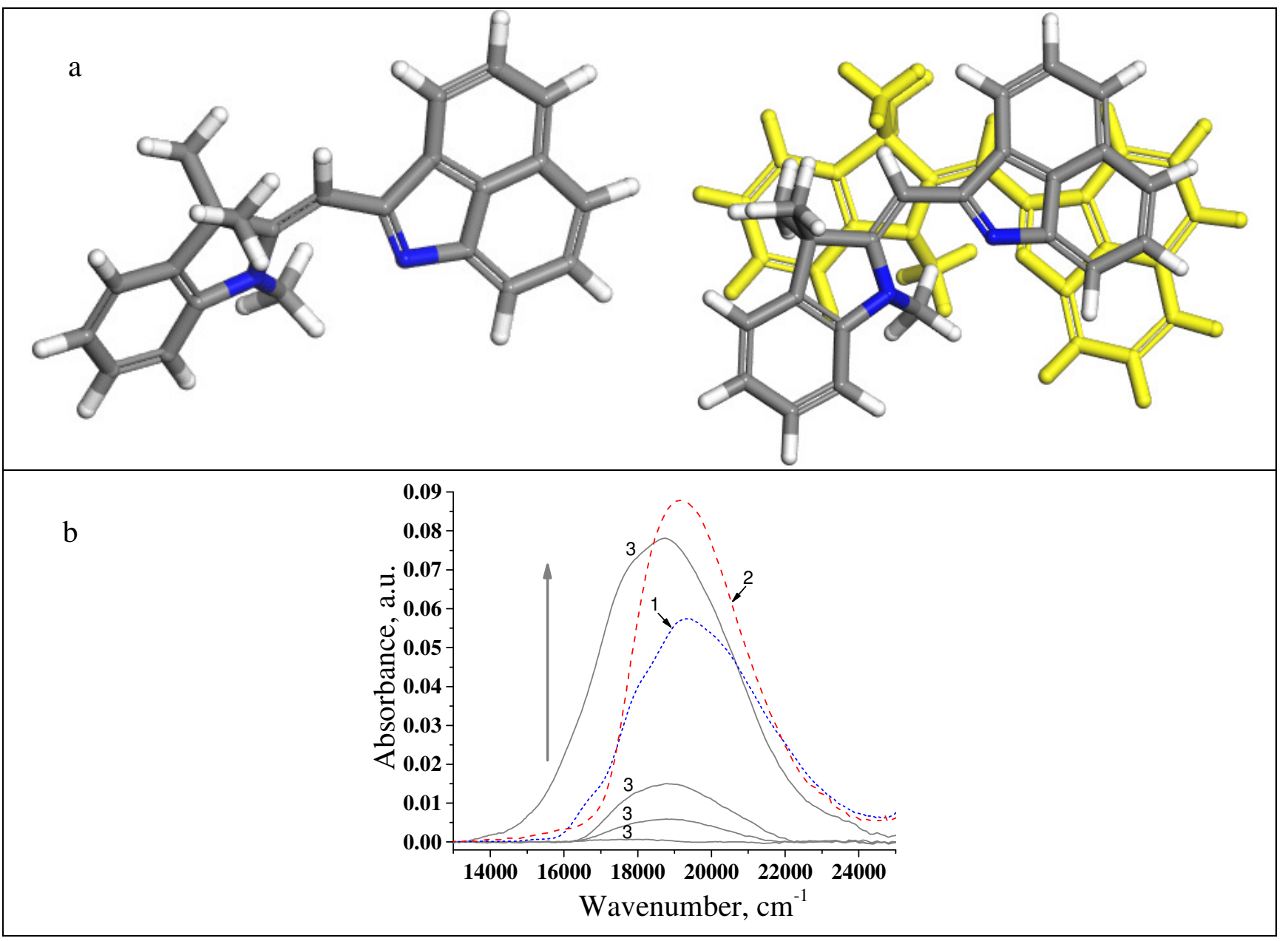

Fig. 5. (a) Simulated structure of In monomer and dimer, (b) electronic absorption spectra of monomer in dioxane solution (blue, 1), dimer in aqueous solution (red, 2) and evaporated film monitored during growth in-situ (grey, 3).

Table 4. Parameters of In monomer and dimer obtained as a result of simulation.

\begin{tabular}{|l|c|c|c|}
\hline \multirow{2}{*}{ Physical quantity } & \multirow{2}{*}{ Monomer } & \multicolumn{2}{|c|}{ Dimer } \\
\cline { 3 - 4 } & & Molecule 1 & \multicolumn{1}{|c|}{ Molecule 2 } \\
\hline Torsion angle & $165.3^{\circ}$ & $170.5^{\circ}$ & 2.29 \\
\hline Dipole moment & 2.49 & 2.24 & \multicolumn{2}{|c}{$40^{\circ}(0.76)$} \\
\hline $\begin{array}{l}\text { Angle between dipole moments of } \\
\text { molecules }(\cos \alpha)\end{array}$ & & & \multicolumn{2}{|c|}{3.3} \\
\hline Dipole moment of dimer & & \\
\hline Distance between molecules $(\AA)$ & & & 3.9 \\
\hline
\end{tabular}

hindrance between approaching molecules, which can affect formation of the tight dimer structure. Simulation showed that the intermolecular distance in the In dimer is relatively large, being $3.9 \AA$, and the overlap of molecules is poor (Fig. 5a, Table 4). As a result, the dimer spectrum was only slightly blue-shifted in respect to the monomer absorption, and films also demonstrated broadened and structureless spectra (Fig. 5b), indicating that the film structure contains high disorder. It should be noted that the above results are similar to those of Py, although the structure of the Py and In molecules are quite different.

\section{Conclusions}

In summary, experimental and simulation results of the aggregate formation of four different monomethincyanine dyes allowed us to derive the major factors responsible for the structural changes both in elementary aggregates, i.e., dimers, and in extended condensed films of these compounds as well. The major parameter is the molecular structure that, dependent on its specific, allows the molecules to approach by different distance and to form different extent of overlap due to the balance of the repulsive and attractive forces, respectively. The 
intermolecular distance and overlap then defines the extent of intermolecular interaction and influences on electronic excitation in the aggregate. Thus, the tentative aggregate structure can be inferred as based on electronic absorption spectra of the species. However, it should be noted that although the main understanding on relation between elementary $\mathrm{H}$ - and J-aggregate structures and their absorption spectra was reached long ago [16], still there is a plenty of room to explain specific aggregate behavior of complex molecules like the studied ones. Particularly, similar stacking of the molecules into the dimer structure was shown to produce different absorption spectra, depending on the mutual overlap and intermolecular distance of the molecules.

\section{Acknowledgements}

We thank to Dr Yu. Slominskii for dyes granting, Dr P. Lytvyn for AFM studies and Dr K. Grytsenko for useful comments.

\section{References}

1. Tan H. Factors affecting molecular self-assembly and its mechanism. Sci. Res. J. 2012. 9, No 1. P. 43-61.

2. Heyne B. Self-assembly of organic dyes in supramolecular aggregates. Photochem. Photobiol. Sci. 2016. 15. P. 1103-1114. DOI: $10.1039 / \mathrm{C6PP00221H.}$

3. Würthner F. Dipole-dipole interaction driven selfassembly of merocyanine dyes: From dimers to nanoscale objects and supramolecular materials. Accounts of Chemical Research. 2016. 49, No 5. 868-876. DOI: 10.1021/acs.accounts.6b00042.

4. Soriano E., Holder C., Levitz A., Henary M. Benz[c,d]indolium-containing monomethine cyanine dyes: Synthesis and photophysical properties. Molecules (Basel, Switzerland). 2016. 21, No 1. P. 23 (1-15).

DOI: 10.3390/molecules21010023.

5. Tolmachev O., Pilipchuk N., Kachkovsky O. et al. Spectral and non-linear optical properties of cyanine bases' derivatives of benzo[c,d]indole. Dyes and Pigments. 2007. 74. P. 195-201.

DOI: 0.1016/j.dyepig.2006.01.048.

6. Sieryk M., Dimitriev O., Doroshenko T., Grytsenko K., Slominski Y. and Kachkovsky O. Aggregation of derivatives of benz[C, D]indole dyes: Effect of the side group and ambient temperatures. 2014 IEEE 34th Intern. Sci. Conf. on Electron. and Nanotechnol. (ELNANO), Kyiv, 2014. P. 223-226. DOI: 10.1109/ELNANO.2014.6873928.

7. Sieryk M., Dimitriev O. Self-assembly of monomethyncyanine and merocyanine dyes: Impact of the intramolecular dipole and substrate used. Front Nanosci Nanotech. 2018. 4, No 2. P. 1-6. DOI: $10.15761 /$ FNN.1000170.

8. Grytsenko K., Doroshenko T., Kolomzarov Yu., Lytvyn O., Serik M., Tolmachev O., Slominski Yu., Schrader S. Growth and optical properties of dye films and dye-in-polymer matrix deposited by vacuum evaporation. Semiconductor Physics, Quantum Electronics \& Optoelectronics. 2010. 13, No 2. P. 177-179.

9. Mukhopadhyay S., Risko C., Marder S.R., Brédas J.-L. Polymethine dyes for all-optical switching applications: A quantum-chemical characterization of counter-ion and aggregation effects on the third-order nonlinear optical response. Chem. Sci. 2012. 3. P. 3103-3112. DOI: 10.1039/C2SC20861J.

10. Tatikolov A.S. Polymethine dyes as spectralfluorescent probes for biomacromolecules. $J$. Photochem. and Photobiol. C: Photochem. Rev. 2012. 13. P. 55-90.

DOI: 10.1016/j.jphotochemrev.2011.11.001.

11. Shapiro B.I. Molecular assemblies of polymethine dyes. Russian Chem. Rev. 2006. 75. P. 433-456.

12. Hestand N., Spano F. Expanded theory of H- and Jmolecular aggregates: The effects of vibronic coupling and intermolecular charge transfer. Chem. Rev. 2018. 118, No 15. P. 7069-7163.

DOI: 10.1021/acs.chemrev.7b00581.

13. Cai K., Xie J., Zhang D., Shi W., Yan Q., Zhao D. Concurrent cooperative J-aggregates and anticooperative H-aggregates. J. Amer. Chem. Soc. 2018. 140, No 17. P. 5764-5773. DOI: $10.1021 /$ jacs.8b01463.

14. Hunter C., Sanders J. The nature of $\pi-\pi$ interactions. J. Amer. Chem. Soc. 1990. 112, No 14. P. 5525-5534.

15. Grytsenko K., Doroshenko T., Kolomzarov Yu. et al. Research on the growth of dye film in vacuum in situ. Proc. SPIE. 2008. 6999. P. 69991Z-6. DOI: $10.1117 / 12.780707$.

16. Kasha M. Energy transfer mechanisms and the molecular exciton model for molecular aggregates. Radiat. Res. 1963. 20. P. 55-64.

DOI: $10.2307 / 3571331$.

\section{Authors and CV}

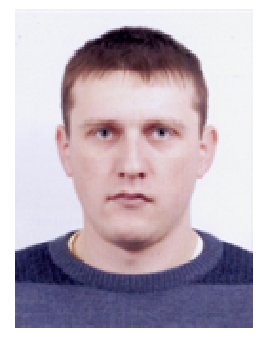

Mykola M. Sieryk, Researcher at the V. Lashkaryov Institute of Semiconductor Physics, NAS of Ukraine. Authored over 6 publication and 2 patents. The area of his scientific interests is optical properties and selforganization of molecular thin films.

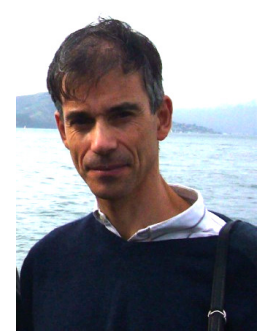

Oleg P. Dimitriev, Senior Research Fellow at the V. Lashkaryov Institute of Semiconductor Physics, NAS of Ukraine. The author of over 100 publications, including 6 patents and 2 books. The area of his scientific interests includes material science of organic and hybrid heterostructures and photophysics of conductive polymers and dyes. 